(1)

CrossMark

\title{
Exercise: shifting fluid and sleep apnoea away
}

\author{
Christopher E. Kline
}

Affiliation: Physical Activity and Weight Management Research Center, Dept of Health and Physical Activity, University of Pittsburgh, Pittsburgh, PA, USA.

Correspondence: Christopher E. Kline, Physical Activity and Weight Management Research Center, Dept of Health and Physical Activity, University of Pittsburgh, 32 Oak Hill Court, Room 227, Pittsburgh, PA 15261, USA.

E-mail: chrisklineapitt.edu

-

@ERSpublications

Exercise may reduce SDB severity through reduction in nocturnal rostral fluid shift http://ow.ly/4nbPZz

Sleep disordered breathing (SDB) is a prevalent sleep disorder $[1,2]$ with a wide array of potential adverse health consequences if left untreated, including cognitive impairment, type 2 diabetes, cardiovascular disease and early mortality [3-6]. The most common type of SDB, obstructive sleep apnoea (OSA), is strongly tied to excess weight [7] and afflicts up to $10 \%$ of adults [2]. Positive airway pressure (PAP) therapy is the most established treatment for OSA and central sleep apnoea (CSA), the other common type of SDB. PAP therapy is highly efficacious at reducing apnoeas and hypopneas during sleep, and its consistent use has been shown to reduce health risk to near non-SDB levels $[8,9]$. However, its efficacy is dependent upon regular use, and long-term adherence to PAP is often unacceptably low [10].

As an adjunctive treatment option or alternative if PAP therapy is not tolerated, weight loss is commonly recommended for overweight/obese adults with OSA [11]. Behavioural lifestyle interventions have been shown to be effective at inducing significant weight loss and OSA severity reduction [12] and have typically focused on dietary modification, with physical activity recommended to increase energy expenditure. However, a consistent, though small, body of literature is emerging to suggest that exercise may have effects on OSA severity independent of any effects on body weight [13]. A recent meta-analysis found that 3-6 months of exercise training reduces the apnoea-hypopnea index (AHI), a standard measure of SDB severity, by $~ 32 \%$ in adults with OSA [14]. Additional small trials have observed AHI reduction following exercise training in adults with CSA $[15,16]$. When also considering its potential benefits on daytime functioning and cardiovascular risk $[17,18]$, exercise may be an attractive adjunct treatment for SDB regardless of whether weight loss is accomplished.

Yet, with such a small body of evidence, much remains unknown regarding the effect of exercise on SDB. For instance, aside from weight loss, few mechanisms that could explain how exercise reduces SDB severity have been explored $[13,19]$. Additionally, it is unknown whether SDB severity improvement occurs only once aerobic exercise-induced training adaptations (e.g. increased peak oxygen consumption $\left(V^{\prime} \mathrm{O}_{2}\right.$ peak)) manifest, which typically takes at least 8 weeks. Research in this issue of the European Respiratory Journal suggests that AHI reduction of $34 \%$ is achievable with as little as 4 weeks of exercise, through attenuation of the nocturnal rostral fluid shift [20].

Although SDB is caused by a variety of weight-dependent and -independent mechanisms [21], lower-limb fluid accumulation (and its subsequent overnight rostral redistribution) has emerged as a contributor to the pathogenesis of SDB [22], especially among adults with significant fluid retention (e.g. heart failure).

Received: April 212016 | Accepted: April 222016

Support statement: Funding was provided by the National Heart, Lung, and Blood Institute, grant K23HL118318 (National Institutes of Health, US Dept of Health and Human Services). Funding information for this article has been deposited with FundRef.

Conflict of interest: None declared.

Copyright OERS 2016 
Gravitational forces lead to the daytime retention of fluid in the interstitial and intravascular compartments of the lower limbs which, upon lying down at night, shifts to the head and upper body. Redistribution to the neck reduces the luminal size of the upper airway and increases its collapsibility, thereby predisposing to OSA. In contrast, nocturnal fluid redistribution to the chest probably leads to pulmonary congestion, leading to CSA via hyperventilation and hypocapnia [22]. Because the primary physiological mechanism preventing lower-limb fluid accumulation is leg skeletal muscle contraction, a highly inactive lifestyle may contribute to SDB severity through this mechanism [19]. MendeLson et al. [20] hypothesised that exercise training would reduce the magnitude of nocturnal rostral fluid shift via engagement of muscle pump activity, thereby lessening the displacement of fluid to the thoracic cavity (for CSA) and neck (for OSA) and reducing AHI.

To test their hypothesis, Mendelson et al. [20] randomised 44 adults with coronary artery disease and moderate to severe SDB to 4 weeks of exercise or a no-treatment control condition. The exercise intervention consisted of $150 \mathrm{~min}$ of moderate-intensity aerobic exercise per week, accomplished by 30 min of walking at $60 \%$ of $V^{\prime} \mathrm{O}_{2}$ peak on 5 days.week ${ }^{-1}$ in both supervised and unsupervised sessions. Before and after the 4-week period, participants underwent maximal exercise testing to evaluate fitness and overnight polysomnography to assess SDB severity. Before and after sleeping, upper-airway cross-sectional area was assessed with acoustic rhinometry, fluid volumes (leg, thoracic and neck) were measured with bioelectrical impedance, and neck and calf circumferences were taken. Of the 34 predominantly male patients who completed the study (17 in each condition), AHI was reduced by $34 \%$ in the exercise group, which was significantly greater than the $4 \%$ reduction observed in the control group. The AHI reduction was accompanied by greater reduction in the overnight change in leg fluid volume and increase in the overnight change in upper-airway cross-sectional area in the exercise group. Importantly, the exercise group did not lose weight or significantly improve their fitness from the 4 -week intervention.

This study's findings confirm a modest effect of exercise on AHI reduction among adults with SDB and suggest that the AHI reduction was due to a reduced nocturnal fluid shift and subsequent retention of upper airway size. The results are similar to those observed by ReDolfi et al. [23] in a randomised crossover trial of eight adults. In this study, 1 week of moderate-intensity walking twice per day for 45 min per session resulted in a $30 \%$ AHI reduction that coincided with a reduction in overnight fluid shift, reduction in nocturnal enlargement in neck circumference, and suppression of nocturnal change in pharyngeal air volume (as measured by magnetic resonance imaging). In addition to the larger sample size, a key advantage of the study by MeNDelson et al. [20] is their examination of a much more realistic exercise dose $\left(150 \mathrm{~min} \cdot \mathrm{week}^{-1}\right.$ versus $\left.630 \mathrm{~min} \cdot \mathrm{week}^{-1}\right)$. Nevertheless, the similarity of study results lends additional credibility to the hypothesis that exercise may reduce SDB severity through attenuating the nocturnal rostral fluid shift.

An important unresolved issue in the study by MENDELSON et al. [20] is whether the effect of exercise on AHI was solely via an acute effect of exercise on the nocturnal rostral fluid shift or whether 4 weeks of exercise training led to additional adaptations that contributed to AHI reduction in addition to the observed reduction in overnight fluid redistribution. If the authors considered the effect of exercise to be driven by its acute effect on lower-limb fluid accumulation, it is unclear why a 4-week intervention was deemed necessary. Notably, KLINE et al. [13] previously observed a $24 \%$ reduction in AHI following 12 weeks of moderate-intensity exercise training; assessment of SDB severity at post-intervention occurred following a day of no exercise, so rostral fluid shift attenuation was a less plausible mechanism. Other mechanisms that may explain the effect of exercise training on SDB include increased respiratory stability through more consolidated and deeper sleep, increased strength and fatigue resistance of the upper airway dilators, and decreased nasal resistance [13]. To date, these mechanisms have been inadequately tested and remain largely speculative.

Despite the promising results from this and other studies on the effect of exercise on SDB, their impact may be limited due to the low long-term compliance to exercise. Few adults meet the public health recommendations for physical activity (i.e. $150 \mathrm{~min} \cdot \mathrm{week}^{-1}$ of moderate-intensity aerobic activity, at least 2 days.week ${ }^{-1}$ of strength-based activity) [24]. Moreover, transitioning to a physically active lifestyle may be even more difficult for adults with SDB, who frequently report daytime fatigue and lack of energy due to their chronically fragmented sleep [25]. Especially since daytime lower-limb fluid accumulation is greatest while seated, a more feasible public health approach may simply be reducing sedentary behaviour (i.e. sitting) rather than requiring moderate-intensity physical activity [26]. Prior research has demonstrated an association between lower daytime sitting duration and a smaller shift in overnight fluid volume, which consequently was associated with lower AHI [27]. Thus, whether breaking up prolonged sitting with standing or other light-intensity activity has an impact on SDB severity merits exploration. Furthermore, because adults can be physically active yet also highly sedentary, a combination of physical inactivity and high sedentary behaviour may place one at especially high risk for SDB. KLINE and HaLL [28] found that community-based adults with a combination of high levels of sedentary time and low physical activity had higher AHI values than all other adults. Moreover, BumAN et al. [29] found that 
television viewing while sitting was associated with being "high risk" for OSA, but that this association was negated among those who were regularly physically active. With television viewing typically undertaken in the evening, the latter study also highlights whether the timing of sedentary behaviour affects SDB severity and whether increasing activity in the hours leading up to bedtime could be particularly beneficial at reducing SDB severity. These possibilities merit inquiry with experimental trials.

In conclusion, Mendelson et al. [20] provide intriguing experimental evidence that a short-term exercise protocol results in significant, albeit modest, reductions in SDB severity that occur alongside a reduction in nocturnal rostral fluid shift despite no change in body weight or fitness. Further work is needed to explore whether similar reductions occur in women, adults without coronary artery disease, and among adults with CSA. Nevertheless, this work should spur additional exploration into how exercise may be utilised in the prevention and management of SDB.

\section{References}

1 Arnardottir ES, Bjornsdottir E, Olafsdottir KA, et al. Obstructive sleep apnoea in the general population: highly prevalent but minimal symptoms. Eur Respir J 2016; 47: 194-202.

2 Peppard PE, Young T, Barnet $\mathrm{JH}$, et al. Increased prevalence of sleep-disordered breathing in adults. Am J Epidemiol 2013; 177: 1006-1014.

3 Vaessen TJ, Overeem S, Sitskoorn MM. Cognitive complaints in obstructive sleep apnea. Sleep Med Rev 2015; 19: 51-58.

$4 \quad$ Lévy P, Bonsignore MR, Eckel J. Sleep, sleep-disordered breathing and metabolic consequences. Eur Respir J 2009; 34: $243-260$.

5 Wang $\mathrm{X}$, Ouyang $\mathrm{Y}$, Wang $\mathrm{Z}$, et al. Obstructive sleep apnea and risk of cardiovascular disease and all-cause mortality: a meta-analysis of prospective cohort studies. Int J Cardiol 2013; 169: 207-214.

6 Mokhlesi B, Ham SA, Gozal D. The effect of sex and age on the comorbidity burden of OSA: an observational analysis from a large nationwide US health claims database. Eur Respir J 2016; 47: 1162-1169.

$7 \quad$ Young T, Peppard PE, Taheri S. Excess weight and sleep-disordered breathing. J Appl Physiol 2005; 99: 1592-1599.

8 Pamidi S, Wroblewski K, Stepien M, et al. Eight hours of nightly continuous positive airway pressure treatment of obstructive sleep apnea improves glucose metabolism in patients with prediabetes. A randomized controlled trial. Am J Respir Crit Care Med 2015; 192: 96-105.

9 Kritikou I, Basta M, Vgontzas AN, et al. Sleep apnoea and the hypothalamic-pituitary-adrenal axis in men and women: effects of continuous positive airway pressure. Eur Respir J 2016; 47: 531-540.

10 Weaver TE, Grunstein RR. Adherence to continuous positive airway pressure therapy: the challenge to effective treatment. Proc Am Thorac Soc 2008; 5: 173-178.

11 Aurora RN, Collop NA, Jacobowitz O, et al. Quality measures for the care of adult patients with obstructive sleep apnea. J Clin Sleep Med 2015; 11: 357-383.

12 Araghi MH, Chen YF, Jagielski A, et al. Effectiveness of lifestyle interventions on obstructive sleep apnea (OSA): systematic review and meta-analysis. Sleep 2013; 36: 1553-1562.

13 Kline CE, Crowley EP, Ewing GB, et al. The effect of exercise training on obstructive sleep apnea and sleep quality: a randomized controlled trial. Sleep 2011; 34: 1631-1640.

14 Iftikhar IH, Kline CE, Youngstedt SD. Effects of exercise training on sleep apnea: a meta-analysis. Lung 2014; 192: 175-184.

15 Servantes DM, Pelcerman A, Salvetti XM, et al. Effects of home-based exercise training for patients with chronic heart failure and sleep apnoea: a randomized comparison of two different programmes. Clin Rehabil 2012; 26: 45-57.

16 Yamamoto U, Mohri M, Shimada K, et al. Six-month aerobic exercise training ameliorates central sleep apnea in patients with chronic heart failure. J Card Fail 2007; 13: 825-829.

17 Kline CE, Ewing GB, Burch JB, et al. Exercise training improves selected aspects of daytime functioning in adults with obstructive sleep apnea. J Clin Sleep Med 2012; 8: 357-365.

18 Kline CE, Crowley EP, Ewing GB, et al. Blunted heart rate recovery is improved following exercise training in overweight adults with obstructive sleep apnea. Int J Cardiol 2013; 167: 1610-1615.

19 Mirrakhimov AE. Physical exercise related improvement in obstructive sleep apnea. Look for the rostral fluid shift. Med Hypotheses 2013; 80: 125-128.

20 Mendelson M, Lyons OD, Yadollahi A, et al. Effects of exercise training on sleep apnoea in patients with coronary artery disease: a randomised trial. Eur Respir J 2016; 48: 142-150.

21 Eckert DJ, White DP, Jordan AS, et al. Defining phenotypic causes of obstructive sleep apnea. Identification of novel therapeutic targets. Am J Respir Crit Care Med 2013; 188: 996-1004.

22 White LH, Bradley TD. Role of nocturnal rostral fluid shift in the pathogenesis of obstructive and central sleep apnoea. J Physiol 2013; 591: 1179-1193.

23 Redolfi S, Bettinzoli M, Venturoli N, et al. Attenuation of obstructive sleep apnea and overnight rostral fluid shift by physical activity. Am J Respir Crit Care Med 2015; 191: 856-858.

24 Centers for Disease Control and Prevention (CDC). Adult participation in aerobic and muscle-strengthening physical activities - United States, 2011. MMWR Morb Mortal Wkly Rep 2013; 62: 326-330.

25 Chervin RD. Sleepiness, fatigue, tiredness, and lack of energy in obstructive sleep apnea. Chest 2000; 118: 372-379.

26 Owen N, Healy GN, Matthews CE, et al. Too much sitting: the population health science of sedentary behavior. Exerc Sport Sci Rev 2010; 38: 105-113.

27 Redolfi S, Yumino D, Ruttanaumpawan P, et al. Relationship between overnight rostral fluid shift and obstructive sleep apnea in nonobese men. Am J Respir Crit Care Med 2009; 179: 241-246.

28 Kline CE, Hall MH. Associations of sedentary time and moderate-vigorous physical activity with sleep-disordered breathing. Sleep 2014; 37: Suppl., A155-A156.

29 Buman MP, Kline CE, Youngstedt SD, et al. Sitting and television viewing: novel risk factors for sleep disturbance and apnea risk? Results from the 2013 National Sleep Foundation Sleep in America Poll. Chest 2015; 147: 728-734. 